\title{
El Desequilibrio en la pareja: la codependencia desde la teoría de interdependencia ${ }^{2}$
}

\author{
Pablo Tonathiu Salcedo Callado \\ PhD. en Psicología \\ Universidad Nacional Autónoma de México, México. \\ Correo electrónico: pablo_tona10@hotmail.com
}

Sofía Rivera Aragón

PhD. en Psicología

Universidad Nacional Autónoma de México, México

Correo electrónico: sofiar1@me.com

\section{Alejandra del Carmen Domínguez Espinosa \\ PhD. en Psicología Social \\ Universidad lberoamericana, México \\ Correo electrónico: alejandra.dominguez@ibero.mx}

Rolando Díaz Loving

Posdoctorado Psicología

Universidad de Texas en Austin, E.U.A

Universidad Nacional Autónoma de México, México

Correo electrónico: rdiazl@unam.mx

Recibido: 29/05/2019

Evaluado: $15 / 07 / 2019$

Aceptado: 19/03/2020

\section{Resumen}

La codependencia se estudia tradicionalmente como rasgo de personalidad, sin embargo, existen sugerencias sobre su posible explicación desde modelos más sociales. Usando el modelo de interdependencia se propone su predicción a través del manejo del conflicto y los estilos de poder. Para ello, participaron 571 adultos $(M=20.20$ años, $D E=1.93$ ) quienes respondieron escalas de manejo del conflicto, estilos de poder y codependencia. Se encontró evidencia a favor del modelo de interdependencia ya que el manejo del conflicto no colaborador en la pareja, y la acomodación y evitación en el codependiente, en conjunto con estilos de poder negativos, son predictores de la codependencia. Se discute la aplicabilidad de la teoría de interdependencia como modelo para explicar la codependencia, así como los beneficios para el entendimiento del inicio y mantenimiento de los patrones codependientes.

Palabras clave Dependencia emocional, relaciones románticas, estilos de poder, manejo del conflicto.

2 Para citar este artículo: Salcedo, C. T., Rivera-Aragón, S., Dominguez-Espinosa. A., \& Díaz-Loving, R. (2021). El Desequilibrio en la pareja: la codependencia desde la teoría de interdependencia. Informes Psicológicos, 21(1), 29-41 http://dx.doi.org/10.18566/infpsic.v21n1a02 


\title{
The imbalance in the couple: codependency from the theory of interdependence
}

\begin{abstract}
Codependency is traditionally studied as a personality trait. However, there are suggestions about its possible explanation from more social models. Using the interdependence model, its prediction is proposed through conflict management and power styles. For this, a study was carried out in which 571 adults participated ( $M=20.20$ years, $S D=1.93$ ), who answered scales of conflict management, power styles and codependency. Evidence was found in favor of the interdependence model, since the management of noncollaborative conflict in the couple, as well as accommodation and avoidance in the codependent, together with negative power styles, are predictors of codependency. The applicability of the interdependence theory as a model to explain codependency is discussed, as well as the benefits for understanding the initiation and maintenance of codependent patterns.
\end{abstract}

\section{Keywords}

\section{Desequilíbrio no casal: A codependência desde a teoria da interdependência}

\section{Resumo}

A codependência é tradicionalmente estudada como um traço de personalidade, porém, existem sugestões sobre sua possível explicação a partir de modelos mais sociais. Utilizando o modelo de interdependência, sua previsão é proposta por meio da gestão de conflitos e estilos de poder. Para isso, participaram 571 adultos ( $(M=20,20$ anos, $D P=1,93)$ que responderam às escalas de gestão de conflitos, estilos de poder $\mathrm{e}$ codependência. Foram encontradas evidências a favor do modelo de interdependência, uma vez que a gestão do conflito não cooperativo no casal e a acomodação e evitação no codependente, juntamente com estilos de poder negativos, são preditores de codependência. A aplicabilidade da teoria da interdependência como um modelo para explicar a codependência é discutida, bem como os benefícios para a compreensão da iniciação e manutenção de padrões codependentes.

Palavras chave Dependência emocional, relacionamentos românticos, estilos de poder, gestão de conflitos. 


\section{ntroducción}

La codependencia (COD) en las parejas se define como el monitoreo constante y atención extrema que una persona pone sobre las necesidades de su pareja descuidando las propias en el proceso (Dear, Roberts \& Lange, 2005; Lampis, Cataudella, Busonera, \& Skowron, 2017; Salcedo, Rivera \& Reyes-Lagunes, 2016). Este patrón de comportamiento se caracteriza por el control interpersonal, el autosacrificio y el daño percibido en la relación, lo que conlleva efectos negativos entre los que se encuentran una alta dependencia emocional, alto afecto negativo y disfunciones relacionales (Marks, Blore, Hine \& Dear, 2012).

Tradicionalmente, la COD se estudia como un rasgo de personalidad que se origina durante la infancia, en donde el abuso o maltrato genera esquemas desadaptativos que se traducen en patrones disfuncionales de relación en la edad adulta (Aguilar, Dinitto, Franklin, \& Lopez-Pilknton, 1991; Chang, 2012; 2016; Fagan-Pryor \& Haber, 1992). Estos patrones se cristalizan en el matrimonio, en donde los estudios sugieren que la COD se mantiene debido a la personalidad de uno de los miembros de la pareja o de ambos [e.g. sus estilos de apego o sus rasgos de personalidad bordelinde o narcisistas] (Dodge, Ward \& Wilkiewitz, 2010; Timmen \& Cermak, 1986; Wells, Glickauf-Hughes \& Brass, 1997). Sin embargo, existe otra aproximación en donde se toma en consideración que las relaciones interpersonales $y$, por extensión, la COD puede explicarse a partir de costos y beneficios percibidos por los miembros de la pareja. La teoría de la interdependencia (Kelley \& Thibaut, 1978) es un marco explicativo para entender la interacción entre dos personas en una relación cercana. Según este modelo si existe balance en los costos y beneficios, esto deriva en satisfacción en la relación, mientras que el desequilibrio genera insatisfacción (Rusbult \& Van Lange, 2008; Sabatelli, 1988). Así pues, desde la teoría de interdependencia el enfoque está en los sucesos presentes y el resultado de las relaciones (funcionamiento o disfunción) puede entenderse a través del análisis de elementos ambientales o de interacción, es decir, una aproximación más social.

Con anterioridad, en la década de 1990, se propuso al modelo de la interdependencia para intentar explicar la COD (Wright \& Wright, 1995). Sin embargo, se trataba de estudios teóricos o sugerencias que carecen de la evidencia empírica. Por lo tanto, es preciso comenzar a probar la capacidad explicativa que el modelo de la interdependencia puede tener sobre los comportamientos codependientes, de modo que se encuentre evidencia que respalde su uso y sus explicaciones para este fenómeno entre las parejas.

Kelley y Thibaut (1978), en su modelo de interdependencia, plantean que cuando una parte de la pareja genera un desequilibrio en la distribución de los costos y beneficios percibidos se crea la dependencia unilateral en donde la persona percibe altos costos al mantener la relación al priorizar la satisfacción de necesidades de su pareja, lo que origina disrupción emocional e insatisfacción, que usualmente llevan a la disolución de la relación (Kelley \& Thibaut, 1978). Sin embargo, en casos poco comunes, la dependencia unilateral no lleva a la disolución de la relación, sino que causa un detrimento progresivo en la 
persona que la experimenta mientras que se agudiza la dependencia emocional a su relación. La COD se podría visualizar como un caso especial de dependencia unilateral donde existen comportamientos de autosacrificio frente a los conflictos y control interpersonal con la intención de proteger a la pareja a toda costa, los mismos que desequilibran los costos y beneficios percibidos en una relación, generando un daño que se experimenta conforme se agudizan estos comportamientos.

Así pues, para probar la aplicabilidad del modelo de interdependencia para la explicación de la COD, podría considerarse que, si la COD es un caso especial de dependencia unilateral, se debería poder predecir a partir de variables que generan desequilibrio en las relaciones de pareja tal como lo postula el modelo de interdependencia. De esta manera, dos variables que afectan el equilibrio de costos y beneficios de una relación son el manejo del conflicto, entendido como el afrontamiento a los problemas en la relación de pareja (Merolla \& Harman, 2018; Righetti, Van Lange, Visserman, Impett, \& Keltner, 2017) y el estilo de poder definido como la forma como un miembro de la pareja obtiene ganancias o satisface sus necesidades en la relación (Bentley, Galliher, \& Ferguson, 2007; Carpenter, 2017).

El conflicto en la pareja para los dependientes emocionales y, por ende, para los codependientes, es fuente de disrupción a nivel psicológico, ya que las situaciones conflictivas intensas pueden llevar a la disolución de una relación que desea mantener a toda costa (Lemos, 2007). De tal modo que el manejo adecuado de los conflictos es indispensable para que una relación se mantenga en equilibrio y pueda permanecer a través del tiempo (Merolla
\& Harman, 2018). Así, sin importar la variedad diferente de estilos que puedan expresarse, se ha propuesto la existencia de dimensiones latentes consistentes en la literatura y las mediciones de este fenómeno: los manejos colaboradores (en posibles dimensiones como estilos negociadores o acomodación) y no colaboradores (en posibles dimensiones como estilos agresivos o apáticos) (Peleg-Koriat, Weimann-Saks, \& Ben-Ari, 2018).

Estudios realizados desde la perspectiva de interdependencia sugieren que, para que una relación permanezca estable incluso si se genera desequilibrio, como es el caso de la dependencia unilateral (la codependencia en este estudio), la persona dependiente debe buscar el manejo del conflicto que garantice la permanencia en la relación (estilos de acomodación ante las necesidades del otro [autosacrificio de las necesidades], o estilos de evitación del problema en cuestión) (Merolla \& Harman, 2018; Righetti et al., 2017) ya frente a un conflicto, los codependientes suelen percibir constantemente que su pareja no colabora en la resolución del problema (Curran, Burke, Young, \& Totenhagen, 2016). De esto, se puede derivar la siguiente hipótesis:

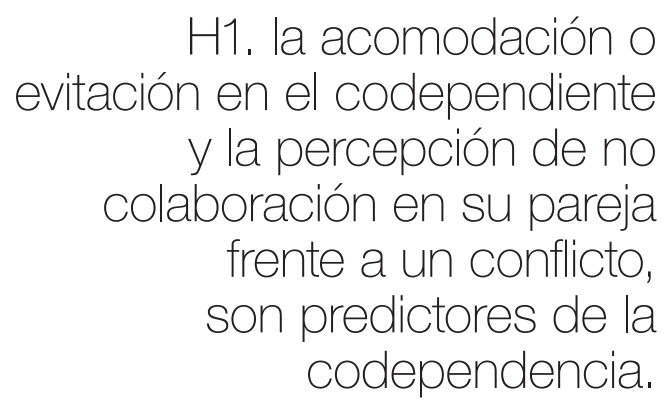

Por otro lado, el ejercicio de poder en la pareja impacta directamente en el equilibrio de la relación, ya que gracias a él se definen los costos y beneficios en 
la relación producto de la satisfacción de necesidades (Bentley et al., 2007). Se ha encontrado que el balance del poder en las relaciones de pareja es fundamental para mantener un equilibrio en los costos y beneficios dentro de ellas (Bentley et al., 2007), y que si este balance se rompe por alguno de los miembros, la relación podría tornarse desequilibrada y poco benéfica para ellos (Carpenter, 2017). Así pues, mientras que unos estilos de poder benefician el equilibrio y la satisfacción mutua de necesidades (e.g. la negociación, democracia o afectuosidad), otros estilos pueden generar desequilibrio al priorizar principalmente los deseos de una de las partes (e.g. el autoritarismo, la agresividad o sumisión) (Carpenter, 2017; Hall \& Knox, 2017). De esta forma, la dimensión negativa de los estilos de poder (estilos que generan desequilibrio) podría predecir la presencia de comportamientos codependientes, de lo que se desprende la siguiente hipótesis:

\section{H2. Los Estilos de poder negativos son predictores de la codependencia.}

En síntesis, evaluar si los estilos de poder o el manejo del conflicto predicen efectivamente la COD podría ayudar a confirmar la aplicabilidad de la teoría de interdependencia para la explicación de este fenómeno, de modo que pueda utilizarse esta teoría como punto de partida para generar intervenciones a partir de variables relacionadas con el funcionamiento de la relación. Por lo tanto, el propósito de esta investigación fue poner a prueba la aplicación de la teoría de la interdependencia para la predicción de la COD.

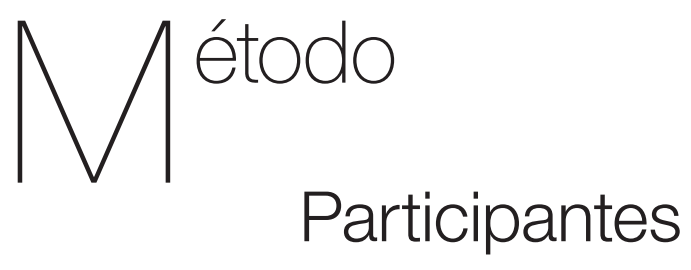

Participaron de manera anónima, confidencial y voluntaria a través de un muestreo no probabilístico accidental, 571 personas (65\% mujeres) de entre 18 y 24 años ( $M=20.20$, $D E=1.93$ ), que se encontraban en una relación de pareja de, al menos, 1 año de duración (con máximo de 8.5 años) y cuya escolaridad era principalmente licenciatura (77\%). Los datos de los participantes fueron recopilados en la Ciudad de México y se excluyeron a los participantes que se encontraran casados, vivieran juntos o tuvieran hijos, debido a que son características que podían modificar el nivel de compromiso y dependencia en una relación. Los datos completos de la muestra se pueden observar en la Tabla 1.

Tabla 1.

Datos sociodemográficos de la muestra.

\begin{tabular}{|c|c|}
\hline Edad & 18 a 24 años $(M=20.20, D E=1.93)$ \\
\hline Edad de la pareja & 16 a 36 años $(M=20.81, D E=3.13)$ \\
\hline \multicolumn{2}{|l|}{ Escolaridad } \\
\hline Secundaria & $14 \%$ \\
\hline Carrera técnica & $3 \%$ \\
\hline Preparatoria & $27 \%$ \\
\hline Licenciatura & $55 \%$ \\
\hline Posgrado & $1 \%$ \\
\hline \multicolumn{2}{|l|}{ Escolaridad de la pareja } \\
\hline Secundaria & $17 \%$ \\
\hline Carrera técnica & $2 \%$ \\
\hline Preparatoria & $29 \%$ \\
\hline Licenciatura & $50 \%$ \\
\hline Posgrado & $2 \%$ \\
\hline \multicolumn{2}{|l|}{ 0cupación } \\
\hline Estudiante & $70 \%$ \\
\hline Empleado & $26 \%$ \\
\hline Inactivo & $4 \%$ \\
\hline \multicolumn{2}{|l|}{ Ocupación de la pareja } \\
\hline Estudiante & $65 \%$ \\
\hline Empleado & $30 \%$ \\
\hline Inactivo & $5 \%$ \\
\hline Duración de la relación en meses & 12 a 102 meses \\
\hline
\end{tabular}




\section{nstrumentos}

Se utilizó el instrumento de Manejo del Conflicto (López-Parra, García \& Rivera, 2010) que está constituido por dos subescalas:

1) Subescala de Manejo del Conflicto del yo (MCY) que evalúa la manera como se afronta el conflicto de pareja, con 32 afirmaciones en formato Likert pictográfico de 5 opciones ( $1=$ totalmente en desacuerdo a $5=$ totalmente de acuerdo) configurada en seis factores: Intolerante $(\alpha=.81)$, Evitante $(\alpha=.80)$, Negociador $(\alpha=.82)$, Afectonegativo $(\alpha=.75)$, Retirada $(\alpha=.63)$ y Acomodación ( $\alpha=.55)$ con los cuales explica el $54.5 \%$ de la varianza. Para evaluar la presencia de las dimensiones propuestas en la investigación (Peleg-Koriat et al., 2018) se condujo un análisis factorial de segundo orden en el que se encontraron dos factores: Factor 1: Evitación (Constituido por los factores: Intolerante, Retirada, Negociador y Evitante) y Factor 2: Acomodación (Constituido por los factores: Acomodación y Afecto negativo), que explican el $61.26 \%$ de la varianza.

2) Subescala de Manejo del conflicto de la pareja (MCP) que evalúa la percepción que se tiene sobre el afrontamiento de la pareja frente a un conflicto. Está conformada por 34 reactivos con 5 opciones de respuesta de tipo Likert pictográfico ( $1=$ totalmente en desacuerdo a $5=$ totalmente de acuerdo) distribuidos en 5 factores: Agresivo-evitante $(\alpha=.90)$, Ataquedefensa $(\alpha=.89)$, Apático $(\alpha=.78)$, No colaborador ( $\alpha=.76)$, Chantaje $(\alpha=.57)$, con los cuales se explica el $57.16 \%$ de la varianza. Al igual que en la subescala anterior se condujo un análisis de segundo orden para encontrar las dimensiones latentes propuestas en la investigación (Peleg-Koriat et al., 2018) en el que se encontraron dos factores de segundo orden, Factor 1: No colaboración (Constituido por los factores: Apático, Ataque-defensa, No colaborador, agresivo) y Factor 2: Chantaje (Constituido por el factor Chantaje), que explican el $65.87 \%$ de la varianza.

\section{Escala de Estilos de Poder}

(EP, Rivera-Aragón \& Díaz-Loving, 2002). Consta de 39 reactivos en un formato Likert pictográfico con 7 opciones de respuesta, distribuidos 8 factores, 3 estilos negativos: Agresivo Evitante $(\alpha$ $=.76)$, Autoritario $(\alpha=.92)$ y Sumiso $(\alpha$ $=.63)$ y 5 estilos positivos: Afectuoso $(\alpha$ $=.95)$, Democrático $(\alpha=.82)$, Tranquiloconciliador $(\alpha=.929)$, Negociador $(\alpha=$ .63) y Laissez-faire $(\alpha=.75)$, a partir de los cuales se explica el $51.4 \%$ de la varianza. Con la finalidad de encontrar los factores latentes globales (positivos y negativos) (Carpenter, 2017; Hall \& Knox, 2017), se exploraron los factores subyacentes en la escala a partir de un análisis factorial de segundo orden, en el que se halló una estructura bifactorial (Estilos positivos que incluyen los Estilos Afectuoso, Democrático, Tranquilo-conciliador y Laissez-Faire y Estilos negativos, que incluyen los estilos Agresivo-evitante, Negociador [con cargas factoriales negativas], Autoritario y Sumiso) a partir de la cual se explica el $65.58 \%$ de la varianza. 
Escala de Codependencia en el noviazgo

(ECDN, Salcedo \& Rivera, en prensa) evalúa los comportamientos típicamente codependientes a través de 9 afirmaciones en un modelo confirmatorio de 3 factores: Control interpersonal, Daño relacional y Autosacrificio, que cuenta con medidas de ajuste aceptables, producto de un análisis factorial confirmatorio: $\mathrm{X}^{2}=$ 33.780, $p=.068, \mathrm{GFI}=.988, \mathrm{CFI}=.993$, RMSEA $=.028$.

\section{Procedimiento}

La aplicación del instrumento se realizó de manera individual y grupal en diferentes zonas geográficas de la Ciudad de México. El acercamiento a los participantes se realizó preguntando si estaban interesados en participar y solicitando un consentimiento para proporcionar sus datos. Posteriormente, se daba lectura a las instrucciones para el llenado del cuestionario y se les aseguraba que sus datos serían totalmente anónimos y confidenciales. Para responder los cuestionarios se explicó la necesidad de que las respuestas fuesen individuales y se solicitó a aquellos que estuviesen en grupo que se separaran para poder responder. Finalmente, el aplicador se distanciaba unos metros para garantizar la autonomía de las respuestas y agradecía cuando los participantes terminaban de responder el cuestionario (lo cual tardaba aproximadamente 25 minutos).

\section{Análisis de datos}

Se llevaron a cabo estadísticos descriptivos e inferenciales para preparar los datos recolectados evaluando la ausencia de autocorrelaciones no superiores a 1.4 (con el estadístico Durbin-Watson), multicolinealidad (a través del índice de diagnóstico y porcentajes de varianza) y la existencia de datos atípicos mayores a 3 o -3 (mediante un análisis de residuos). Para finalmente, realizar análisis de regresión múltiple por el método enter para responder las hipótesis de investigación las cuales ponen a prueba la predicción de la codependencia en función de los factores del manejo del conflicto (del yo y la pareja) y de los factores de los estilos de poder.

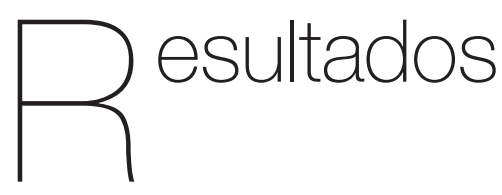

Después de ejecutar los análisis de frecuencias, autocorrelaciones, multicolinealidad y valores atípicos, se eliminaron 4 casos (1 dato perdido y 3 casos atípicos [57, 422 y 434]), por lo que se trabajó con 466 casos con los que se ejecutaron las pruebas de regresión lineal múltiple en donde se encontraron modelos significativos para los tres factores de la COD.

En la Tabla 2 se muestra el detalle del modelo de regresión para el factor Control interpersonal de la COD. En él se observa como predictores significativos al estilo del manejo del conflicto de la pareja a través del chantaje, así como los estilos del manejo del conflicto del yo de evitación y acomodación y los estilos de poder negativos. 
Tabla 2.

Predictores del factor control interpersonal de la codependencia

\begin{tabular}{lccc}
\hline \multirow{2}{*}{ Variables predictoras } & B & \multicolumn{2}{c}{ IC 95\% } \\
\cline { 3 - 4 } & & Inferior & Superior \\
\hline No colaboración (MCP) & .08 & -.01 & .19 \\
Chantaje (MCP) & $.10^{*}$ & .02 & .14 \\
Evitación (MCY) & $.14^{* *}$ & .01 & .05 \\
Acomodación (MCY) & $.17^{* *}$ & .04 & .11 \\
Estilos de poder negativos & $.13^{* *}$ & .01 & .04 \\
Estilos de poder positivos & .03 & -.01 & .01 \\
$R^{2}$ & .13 & & \\
$F$ & $13.39^{* *}$ & & \\
\hline
\end{tabular}

Nota: $\mathrm{MCP}=$ Manejo del conflicto de la pareja, $\mathrm{MCY}=$ Manejo del conflicto del yo, $\mathrm{n}=566$,

IC $95 \%=$ Intervalo de confianza de Beta de $95 \%,{ }^{*} p \leq .05,{ }^{* \star} p \leq .01$.

En la Tabla 3 se muestra el detalle del modelo de regresión para el factor Daño relacional de la COD. Se observan, como predictores significativos, los estilos del manejo del conflicto del yo de evitación y acomodación y los estilos de poder negativos y los estilos de poder positivos.

Tabla 3.

Predictores del factor daño relacional de la codependencia

\begin{tabular}{lccc}
\hline \multirow{2}{*}{ Variables predictoras } & B & \multicolumn{2}{c}{ IC 95\% } \\
\cline { 3 - 4 } & & Inferior & Superior \\
\hline No colaboración (MCP) & .07 & -.01 & .16 \\
Chantaje (MCP) & .01 & -.05 & .05 \\
Evitación (MCY) & $.18^{* *}$ & .02 & .05 \\
Acomodación (MCY) & $.16^{* *}$ & .04 & .09 \\
Estilos de poder negativos & $.18^{* *}$ & .01 & .04 \\
Estilos de poder positivos & $-.17^{* *}$ & -.03 & -.01 \\
$R^{2}$ & .23 & & \\
$F$ & $27.49^{* *}$ & & \\
\hline
\end{tabular}

Nota: $\mathrm{MCP}=$ Manejo del conflicto de la pareja, $\mathrm{MCY}=$ Manejo del conflicto del yo, $\mathrm{n}=566$,

IC $95 \%=$ Intervalo de confianza de Beta de $95 \%,{ }^{\star} \mathrm{p} \leq .05,{ }^{\star \star} \mathrm{p} \leq .01$.

Finalmente, en la Tabla 4 se muestra el detalle del modelo de regresión para el factor Sumisión de la COD. En este modelo obtuvieron como predictores significativos el estilo del manejo del conflicto de la pareja no colaborador, así como el estilo del manejo del conflicto del yo de acomodación y los estilos de poder negativos. 
Tabla 4.

Predictores del factor autosacrificio de la codependencia

\begin{tabular}{lccc}
\hline \multirow{2}{*}{ Variables predictoras } & B & \multicolumn{2}{c}{ IC 95\% } \\
\cline { 3 - 4 } & & Inferior & Superior \\
\hline No colaboración (MCP) & $.18^{* \star}$ & .11 & .3 \\
Chantaje (MCP) & .08 & -.01 & .11 \\
Evitación (MCY) & -.09 & -.04 & .12 \\
Acomodación (MCY) & $.26^{* *}$ & .07 & .13 \\
Estilos de poder negativos & $.14^{* *}$ & .01 & .04 \\
Estilos de poder positivos & -.04 & -.01 & .01 \\
$R^{2}$ & .14 & & \\
$F$ & $14.82^{* *}$ & & \\
\hline
\end{tabular}

Nota: $\mathrm{MCP}=$ Manejo del conflicto de la pareja, $\mathrm{MCY}=$ Manejo del conflicto del yo, $\mathrm{n}=566$,

IC $95 \%=$ Intervalo de confianza de Beta de $95 \%,{ }^{*} p \leq .05,{ }^{* *} p \leq .01$.

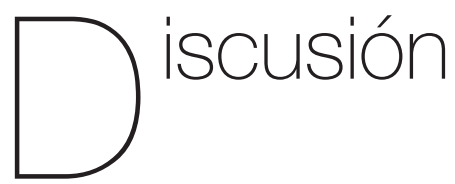

En esta investigación se postuló que la COD podría explicarse aplicando la perspectiva de interdependencia. Para ello, debería entenderse la COD como una forma especial de dependencia unilateral en la cual existe un desequilibrio en los costos y beneficios en la relación de pareja. Para probar estas suposiciones se hipotetizó que estilos de poder negativos y manejos del conflicto de acomodación y evitación (en el codependiente) y no colaboradores (percibidos en su pareja) deberían ser predictores de la COD al ser variables que generan desequilibrio en las relaciones. En los resultados, se encontró evidencia a favor de las hipótesis planteadas, de modo que se puede afirmar que la teoría de la interdependencia puede ser un modelo teórico aplicable para la explicación de la COD.
En general, en los tres modelos de regresión se encontró evidencia a favor de las hipótesis, misma que respalda que la COD puede ser entendida como una dependencia unilateral, al mostrar que el control interpersonal, el daño en la relación y el autosacrificio que componen a la COD pueden ser predichos efectivamente cuando la persona codependiente observa que su pareja no colabora frente al conflicto, acomodándose a la resolución o evitando los problemas, practicando manejos del conflicto que se han asociado a la permanencia de relaciones a través del tiempo y el desequilibrio de la relación de pareja (Curran et al., 2016; Merolla \& Harman, 2018; Peleg-Koriat et al., 2018; Righetti et al., 2017). Al mismo tiempo que ejerce su poder en estilos negativos que facilitan el control interpersonal y favorecen el desequilibrio de los costos y beneficios en una relación a su favor tales como el autoritarismo, la agresividad o la sumisión (Bentley et al., 2007; Carpenter, 2017; Hall \& Knox, 2017).

En particular, la evidencia encontrada sobre la dinámica frente al conflicto 
concuerda con la descripción de la dependencia unilateral propuesta desde la teoría de interdependencia. En esta descripción se dice que el dependiente unilateral es una persona que descuida sus necesidades en la relación en pro del cuidado de su pareja o su relación, priorizando los beneficios a largo plazo, aunque ello involucre una percepción menor de beneficios a corto plazo $(\mathrm{Ke}-$ lley \& Thibaut, 1978; Merolla \& Harman, 2018). De esta forma, como predictores de los tres factores de la COD, se encontró consistentemente el manejo del conflicto de acomodación el cual opera como predictor para todos los factores, seguido del manejo por evitación como predictor del control interpersonal y el daño en la relación. Estos dos estilos permiten al codependiente satisfacer las necesidades de su pareja al momento del conflicto, logrando una resolución rápida en el caso de la acomodación o una percepción de daño menor en el caso de la evitación, lo que posiblemente permite que la relación de pareja continúe incluso si existe desequilibrio (Merolla \& Harman, 2018; Righetti et al., 2017).

Así mismo, en cuanto al ejercicio de poder, se propuso que formas negativas de practicar el poder pueden llevar al desequilibrio de una relación de pareja y generar dependencia unilateral (Bentley et al., 2007; Carpenter, 2017; Hall \& Knox, 2017), lo cual haría a los estilos de poder negativos predictores de la COD según las hipótesis planteadas. Al respecto, se encontró que los estilos negativos de poder son predictores para los tres factores de la COD. Así pues, la práctica de estilos de poder negativos que incluyen comportamientos que van desde el autoritarismo hasta la posible sumisión (Bentley et al., 2007; Carpenter, 2017) están enfocados en controlar o manipular los comportamientos de la pareja generando desequilibrio en los costos y beneficios para una de las partes en la relación, que podría llevar a la dependencia unilateral en forma de COD.

Poder usar la teoría de la interdependencia para la predicción de la COD, facilita el acercamiento a sus posibles causas situacionales, propias de la interacción y no de la personalidad del individuo, nutriendo el conocimiento acerca su aparición y funcionamiento más allá de los modelos tradicionales que suponían su origen en el estudio retrospectivo de la infancia (Fagan-Pryor \& Haber, 1992) o el estudio de la personalidad del codependiente (Dodge et al., 2010; Timmen \& Cermak, 1986). El uso del modelo de la interdependencia aplicado a la COD puede proveer de elementos para diseñar intervenciones centradas en la modificación de comportamientos de interacción, en función de prevenir la aparición de patrones codependientes en alguna de las personas involucradas en la relación.

\section{Limitaciones}

La evidencia obtenida en este estudio debe tomarse como un acercamiento al desequilibrio en la relación de pareja como predictor de la COD, lo cual, a su vez, implica la posibilidad de aplicar el modelo de interdependencia a la explicación de este fenómeno. Para resultados más concluyentes acerca de estás hipótesis es necesario solventar algunas limitaciones. La aproximación de este estudio hacia el fenómeno del desequilibrio se realizó a partir de instrumentos de autoinforme, los cuales tienen una limitación importante debido a la naturaleza retrospectiva 
de la información que se puede obtener de ellos. Un siguiente paso en la investigación sería el diseño y ejecución de experimentos a partir de una perspectiva diádica, desde la cual pueda observarse claramente la interacción de la pareja y los resultados de la misma como un proceso de interacción en el presente.

A modo de conclusión, es importante comenzar a generar explicaciones integrales del fenómeno de la COD, de modo que puedan dilucidarse las variables situacionales de la relación de pareja, así como las variables asociadas al estilo de personalidad de cada uno de sus miembros que intervienen en la aparición y mantenimiento de este patrón negativo de relación.

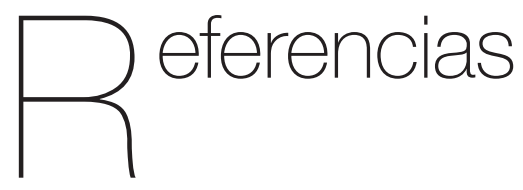

Aguilar, M. A., Dinitto, D. N., Franklin, C., \& Lopez-Pilknton, B. (1991). MexicanAmerican Families: A psychoeducational approach for addressing chemical dependency and codependency. Child and Adolescent Social Work, 8(4), 309-327. DOI: 10.1007/BF00756300.

Bentley, C. G., Galliher, R. V., \& Ferguson, T. J. (2007). Associations among aspects of interpersonal power and relationship functioning in adolescent romantic couples. Sex Roles, 57(7-8), 483-495. https://doi. org/10.1007/s11199-007-9280-7

Carpenter, C. J. (2017). A Relative Commitment Approach to Understanding Power in
Romantic Relationships. Communication Studies, 68(1), 115-130. https://doi.org/10.1 080/10510974.2016.1268639

Chang S-H. (2012). A cultural perspective on codependency and its treatment. Asia Pacific Journal of Counselling and Psychotherapy, 3(1), 50-60. DOI: 10.1080/21507686.2011.620973

Chang, S-H. (2016). Testing a model of codependency for college students in Taiwan based on Bowens concept of differentiation. International Journal of Psychology, 53(2), 107-116. DOI: 10.1002/ ijop.12271.

Curran, M. A., Burke, T. J., Young, V. J., \& Totenhagen, C. J. (2016). Relational Sacrifices about Intimate Behavior and Relationship Quality for Expectant Cohabitors. Marriage and Family Review, 52(5), 442-460. https://doi.org/10.1080/014 94929.2015 .111322

Dear, G. E., Roberts, M. C. \& Lange, L. (2005). Defining codependency: A thematic analysis of published definitions. In S. P. Shohov (Ed). Advances in psychology research (pp. 189-205). Hauppauge, NY, US: Nova Science Publishers.

Dodge, R., Ward, S., \& Witkiewitz K. (2010) Psychosocial Variables as Mediators of the Relationship Between Childhood History of Emotional Maltreatment, Codependency, and Self-Silencing. Journal of Aggression, Maltreatment \& Trauma, 19(2), 159-179, DOI: 10.1080/10926770903539375

Fagan-Pryor, E. \& Haber, C. L. (1992). Codependency: another name for Bowens undifferentiated self. Perspective in Psychiatric Care, 28(4), 24-29. DOI: 10.1111/ j.1744-6163.1992.tb00389.x 
Hall, S. S., \& Knox, D. (2017). Perceived relationship power in emerging adults' romantic relationships. Journal of Family Studies, O(0), 1-12. https://doi.org/10.1080/ 13229400.2016 .1269660

Kelley, H. H. \& Thibaut, J.W. (1978). Interpersonal relations: a theory of interdependence. New York: Wiley.

Lampis, J., Cataudella, S., Busonera, A., \& Skowron, E. A. (2017). The role of differentiation of self and dyadic adjustment in predicting codependency. Contemporary Family Therapy, 39(1), 62-72.

López-Parra, M. S., García, M. M. y Rivera, A. S. (2010). Cómo se maneja el conflicto en el noviazgo. Trabajo presentado en el congreso de la Sociedad Mexicana de Psicología. Universidad Iberoamericana. México, D.F.

Lemos, M. (2007). Distorsiones cognitivas en personas con dependencia emocional. Informes Psicológicos, 9(2), 55-69. https:// doi.org/10.1093/jaarel/52.2.411-a

Lindley, N. \& Hammer, E. (1999). Codependency: Predictors and psychometric issues. Journal of Clinical Psychology, 55(1), 59-64. DOI: 10.1002/ (SICI)1097-4679(199901)55:1<59::AIDJCLP5>3.0.CO;2-M

Marks, D. A., Blore, L. R., Hine, W. D. \& Dear, E. G. (2012). Development and validation of a revised measure of codependency. Australian Journal of Psychology, 64(3), 119127. DOl: 10.1111/j.1742-9536.2011.00034.x

Merolla, A. J., \& Harman, J. J. (2018). Relationship-Specific Hope and Constructive Conflict Management in
Adult Romantic Relationships: Testing an Accommodation Framework. Communication Research, 45(3), 339-364. https://doi.org/10.1177/0093650215627484

Peleg-Koriat, I., Weimann-Saks, D., \& BenAri, R. (2018). The Relationship between Power Perception and Couple's Conflict Management Strategy. Journal of Couple and Relationship Therapy, 17(3), 227-249. https://doi.org/10.1080/15332691.2017.139 9847

Righetti, F., Van Lange, P. A. M., Visserman, M. L., Impett, E. A., \& Keltner, D. (2017). It's the motive that counts: Perceived sacrifice motives and gratitude in romantic relationships. Emotion, 18(5), 625-637. https://doi.org/10.1037/emo0000344

Rivera-Aragón, S. \& Díaz-Loving, R. (2002). La cultura del Poder en la Pareja. Facultad de Psicología, UNAM. México: Porrúa.

Rusbult, C., \& Van Lange, P. (2008). Why we need interdependence theory. Social and personality psychology compass, 2(5), 20049-2070. DOl: 10.1111/j.1751-9004.2008.00147.x

Sabatelli, R. (1988). Exploring Relationship Satisfaction: A Social Exchange Perspective on the Interdependence between Theory, Research, and Practice. Family Relations, 37(2), 217-222. DOI: 10.2307/584323

Salcedo, P., Rivera, S., \& Reyes-Lagunes, I. (2016). La conceptuación de la codependencia del noviazgo. En ed; Diaz-Loving, Reyes-Lagunes, RiveraAragon, Hernandez-Sanchez \& GarcíaFalconi (2016). Aportaciones actuales de la psicología social. AMEPSO, ISBN: 978-607-96539-4-1. 
Timmen L. \& Cermak M.D. (1986). Diagnostic Criteria for Codependency. Journal of Psychoactive Drugs, 18(1), 15-20. DOI: 10.1080/02791072.1986.10524475

Wells, C., Glickauf-Hughes, \& Brass, K. (1997). The Relationship of Co-Dependency to Enduring Personality Characteristics. Journal of College Student Psychotherapy, 12(3), 25-38. DOI: 10.1300/J035v12n03_03
Wright, P. H., \& Wright, K. D. (1995). Codependency: Personality syndrome or relational process? In Understanding Relationship Processes Series, Vol. 5. Confronting relationship challenges. (pp. 109-128). https://doi. org/10.4135/9781483327181.n5 